Document downloaded from:

http://hdl.handle.net/10251/76326

This paper must be cited as:

Giner-Bosch, V.; San Matías Izquierdo, S.; Mónica Clemente-Císcar; Carrión García, A. (2016). Optimal design of pre-control plans. Quality and Reliability Engineering International. 32(2):623-634. doi:10.1002/qre.1777.

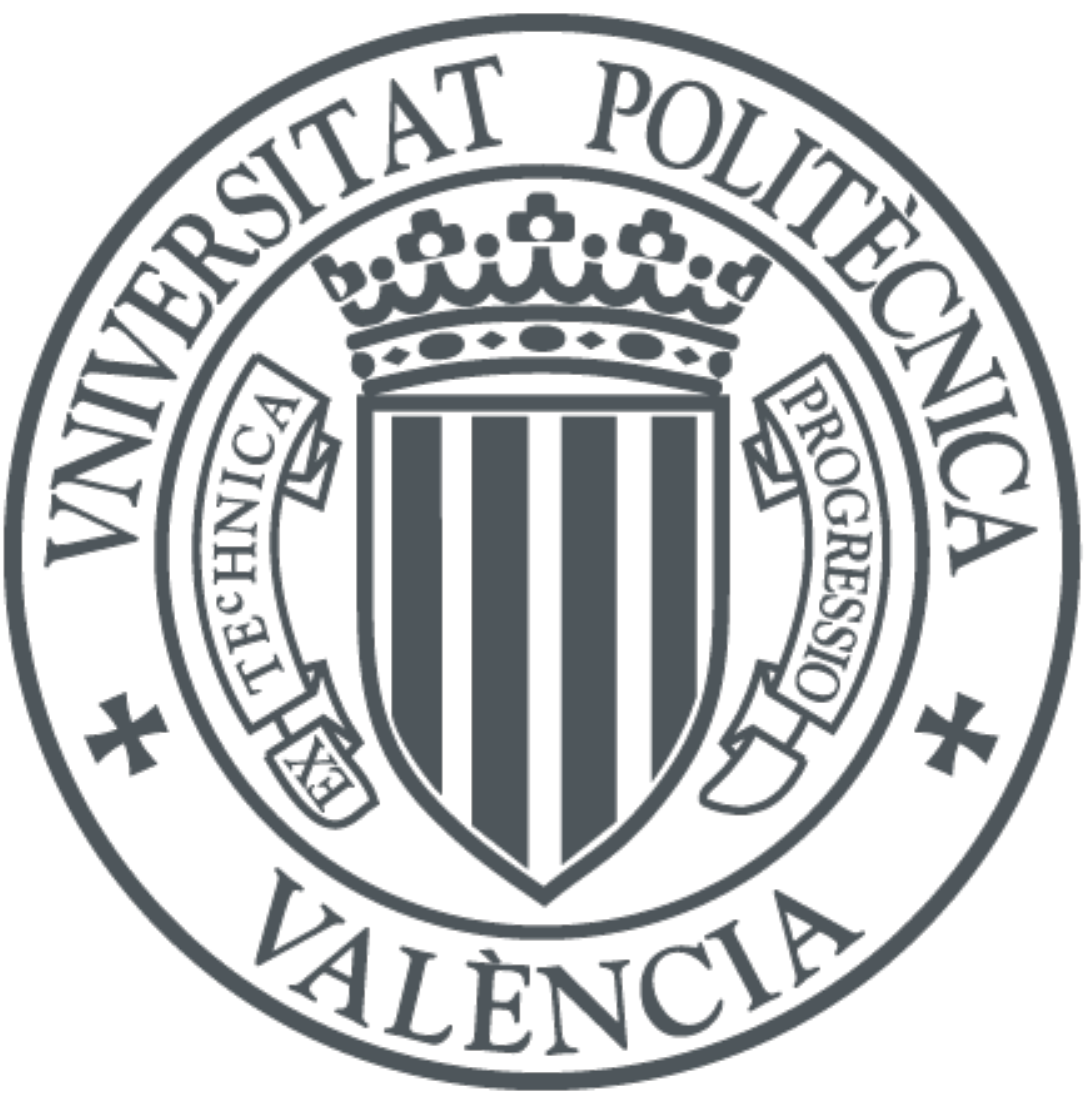

The final publication is available at

http://dx.doi. org/10.1002/qre.1777

Copyright Wiley

Additional Information 


\title{
Optimal design of pre-control plans
}

\author{
Vicent Giner-Bosch ${ }^{\mathrm{a}, *}$, Susana San Matías ${ }^{\mathrm{b}}$, Mónica Clemente-Císcar ${ }^{\mathrm{a}}$, \\ Andrés Carrión ${ }^{\mathrm{a}}$ \\ ${ }^{a}$ Centro de Gestión de la Calidad y el Cambio, Universitat Politècnica de València, \\ Camí de Vera s/n, València 46022, Spain. \\ ${ }^{b}$ Departamento de Estadística e Investigación Operativa Aplicadas y Calidad, \\ Universitat Politècnica de València, Camí de Vera s/n, València 46022, Spain.
}

\begin{abstract}
Pre-control is a quality tool for quick set-up approvals, especially used in short-run processes. It is based on specifications instead of on the natural variability of the process and uses cumulative counts in order to yield a conclusion. Its main drawbacks are a high false alarm rate and a low power to detect process deviations, under certain circumstances. These issues can be addressed by making the technique more flexible, as shown in previous works. In this paper, we introduce a Mathematical Programming approach in order to optimally determine the value of the pre-control parameters, so that it can meet the user's requirements while minimizing the sample size of the technique. We propose and develop a mathematical model for optimal pre-control and perform some numerical experiments in order to show its effectiveness.
\end{abstract}

Keywords: pre-control, set-up approval, process capability, Mixed-Integer Nonlinear Programming, Optimization

\footnotetext{
*Corresponding author. Tel.: +34 9638774 90; Fax: +34 963877499.

Email addresses: vigibos@eio.upv.es (Vicent Giner-Bosch), mclement@eio.upv.es (Mónica Clemente-Císcar), acarrion@eio.upv.es (Andrés Carrión)

Preprint submitted to Quality and Reliability Engineering International November 27, 2014
} 


\section{Introduction}

\subsection{Background and motivation}

Pre-control is a quality control technique originally aimed at quickly assessing the ability of a process to produce units within specifications. It checks individual observations against the specification limits and the socalled pre-control limits, and uses cumulative count information in order to reach to a conclusion. It is a fast, easy to apply tool, and thus is considered to be particularly useful for short-run manufacturing processes.

\subsubsection{Classical pre-control}

Pre-control was initially designed by Satterthwaite ${ }^{1}$ and later revisited by Shainin and Shainin ${ }^{2}$. We will now describe the technique in its original form, henceforth classical pre-control.

In pre-control, the specification range is divided into four areas of equal width, the two central ones being referred to as the green zone. The areas within specifications but outside the green zone are called the yellow zone, and the areas outside the specification range are called the red zone (see figure 1). The values limiting the green zone are referred to as pre-control limits. This zone definition applies to the case of two-sided symmetric specifications, which is the one that this paper deals with. Likewise, each sampled unit is classified as green, yellow or red, depending on the zone it lies in.

When applied as a set-up approval or initial qualification procedure, pre-control operates in the following way: Successive units are sampled until 5 consecutive green units are obtained (and in this case the process is said to be qualified or accepted) or 2 consecutive yellow ones or 1 red one are observed (and in this case an alarm is raised to stop and revise the process). Notice that in this qualification phase or stage the total number of units to be sampled is not previously set or fixed, because any time a series of greens or yellows is broken, the count of consecutive greens or yellows, respectively, is restarted.

After the process is qualified and normal production starts, one unit is periodically sampled in order to decide if the process remains unchanged, 


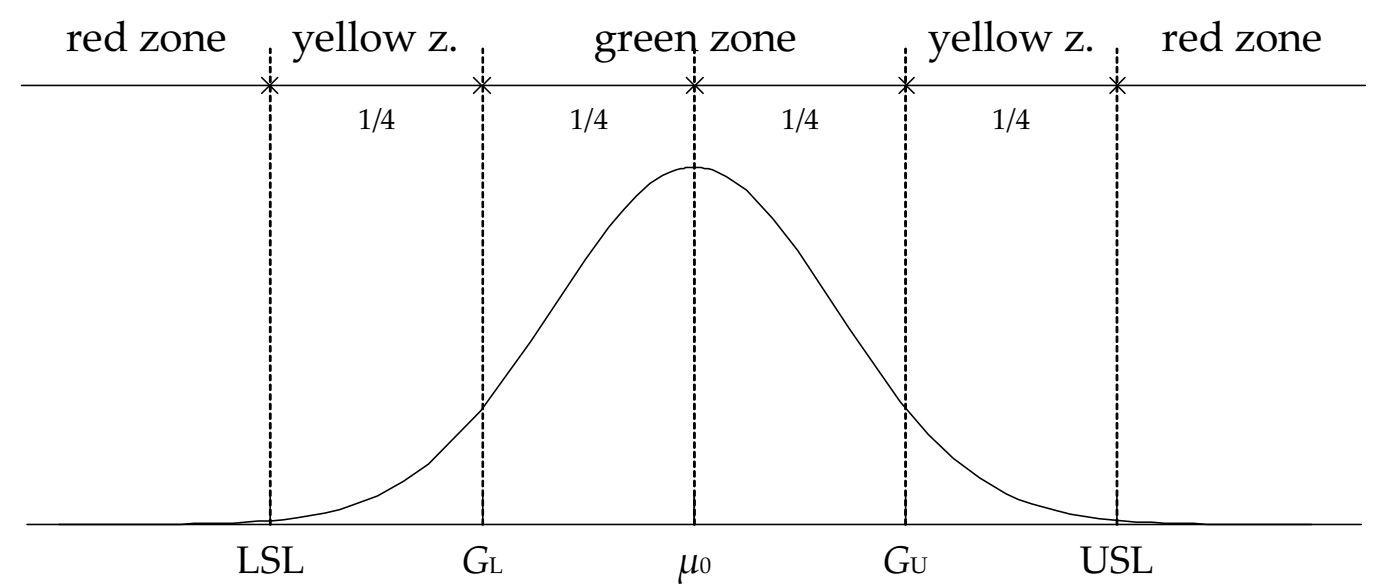

Figure 1: Classification procedure in classical pre-control, for an in-control normal process with potential capability $C_{\mathrm{p}}=1$.

according to the following rule: If that unit is green, then the process is considered to be right, and nothing has to be done until the next scheduled sampling; if it is red, then the process has to be stopped and revised; and if the unit is yellow, then a second unit is sampled and, if that unit is not green, then the process is also stopped and revised. Any time the process is stopped for revision, the qualification phase of pre-control has to be performed before restarting normal production.

As said before, this is a simple, fast procedure that neither requires previous estimation of the variability of the process, nor plotting the data, but only implementation of a reliable way to classify units into green, yellow and red ones.

The main drawback of classical pre-control is, in fact, that it does not take into account the natural variability of the process, and this can lead to undesired situations, such as rejecting a process with a high potential capability which is producing parts within specifications but is not centered (and, thus, yellow units are very likely to appear). On the other hand, when considered as a test on the deviation in the process mean (see Section 2.2 later for further explanation), classical pre-control also shows too high a false alarm rate in centered processes with low 
capability, and a low power to detect relative small mean shifts in highly capable processes (see the notation Section 2.1 later for a formal definition of the potential capability index and other terms that will be used in this paper).

Several attempts existing in the literature to enhance the performance of the classical technique are cited later in Section 1.2.

\subsubsection{Optimization in quality control}

In this paper we propose using Optimization techniques in order to improve the performance of pre-control. In recent decades, Operations Research has been increasingly applied to the optimal design of quality control tools ${ }^{3-5}$, especially when using an economic approach ${ }^{6}$, the adoption of heuristic strategies such as genetic algorithms in particular being quite frequent ${ }^{7-11}$.

The advantages of applying Optimization techniques to quality control and improvement are the same as in other fields: obtaining better tools, better knowledge and control of the system, and the ability to make better decisions.

\subsection{Previous research}

Salvia ${ }^{12}$ and Gruska and Heaphy ${ }^{13}$ propose different modifications to classical pre-control, which are later reviewed by Steiner ${ }^{14}$, who also proposes three alternatives, all of them aimed at improving the performance of pre-control in the regular production stage. In particular, the proposal of Gruska and Heaphy redefines pre-control limits so that they are directly related to the standard deviation of the process when it is under control. In this way, information about the natural variability of the process is incorporated into the pre-control scheme. In simplified pre-control, one of his three proposals, Steiner goes one step further and suggests parametrizing the width of the green zone. All these alternatives try to improve the performance of the classical technique by also slightly increasing the number of pieces to be sampled in the regular phase.

Družovec et al. ${ }^{15}$, San Matías et al. ${ }^{16}$ and Cox et al. ${ }^{17}, 18$ also propose some modifications to pre-control, focusing on the qualification stage this 
time. Družovec et al. heavily modify the definition of the green, yellow and red zones, so that the units within the specification range but close to its limits are classified as red instead of yellow. They also completely redefine the qualification procedure in such a way that the sample size is fixed to a maximum of 6 units.

San Matías et al. suggest parametrizing the qualification phase of precontrol by letting the user choose the number of consecutive green units which are needed to qualify the process, the number of consecutive yellow units required to stop and revise the process and the width of the green zone, in such a way that the procedure meets some given requirements in terms of the false alarm rate and the power to detect unacceptable deviations. We will look further at this proposal in Section 3.

Finally, the work by Cox et al. also comes back to the idea of adjusting the width of the green zone, in this case ensuring that a maximum of $2 \%$ false alarm risk is observed. Apparently, they simultaneously refer to both the probability of adjusting an on-target process (with regards to the mean) and the chance of classifying a capable process as incapable.

It is worth to remark that nearly all the modifications of pre-control above mentioned implicitly or explicitly treat it as a tool for detecting shifts in the mean of the process (see Section 2.2), assuming that the natural variability is known or can be estimated.

\subsection{Aims and structure}

In this paper, the work of San Matías et al. ${ }^{16}$, namely generalized precontrol, is taken as a starting point for our proposal. An Optimization approach will be given to this modification of the qualification phase of the classical technique. Instead of letting the user choose the values for the parameters of generalized pre-control, a Mathematical Programming model will be built in order to determine the best setting for these parameters, with regards to the objective of minimizing the expected sample size of the qualification procedure. As a result, the user will be able to obtain a pre-control plan which is certain to meet his/her requirements using the minimum number of units on average. 
The rest of this paper is structured as follows. In Section 2 some notation and a formal framework for our work are given. Section 3 describes the above mentioned proposal of San Matías et al. ${ }^{16}$ in detail. The mathematical model for the optimal determination of the pre-control parameters will be developed in Section 4. Then, we present some numerical experiments showing the effectiveness of our proposal in Section 5. Finally, conclusions are derived in Section 6.

\section{Notation and preliminaries}

\subsection{Basic notation}

In this work, a quality characteristic $X$ following a normal distribution with mean $\mu$ and standard deviation $\sigma$ is assumed to be monitored. The target values for the parameters of the distribution are denoted by $\mu_{0}$ and $\sigma_{0}$.

The specification limits are denoted by LSL and USL (for lower and upper specification limits, respectively), with LSL $<$ USL. The lower and upper pre-control limits are represented by $G_{L}$ and $G_{U}$, respectively. The width of the green zone will is denoted by $W_{\mathrm{G}}$. Both the specification range and the green zone are supposed to be centered on $\mu_{0}$, that is to say, the following relationships hold:

$$
\begin{aligned}
\mu_{0} & =\frac{\mathrm{USL}+\mathrm{LSL}}{2}, \\
G_{\mathrm{L}} & =\mu_{0}-\frac{W_{\mathrm{G}}}{2}, \\
G_{\mathrm{U}} & =\mu_{0}+\frac{W_{\mathrm{G}}}{2} .
\end{aligned}
$$

Bear in mind that, in classical pre-control, $W_{\mathrm{G}}=(\mathrm{USL}-\mathrm{LSL}) / 2$, but in the proposal by San Matías et al. ${ }^{16}$ this is no longer necessarily true, as the width of the green area is allowed to vary.

We also use a common index regarding the ability of the process to produce units within specifications, that is, the capability of the process 
-more precisely, the potential capability index $C_{\mathrm{p}}$, defined as follows:

$$
C_{\mathrm{p}}=\frac{\mathrm{USL}-\mathrm{LSL}}{6 \sigma} .
$$

The probabilities of obtaining a green, yellow or red unit will be represented by $p_{\mathrm{g}}, p_{\mathrm{y}}$ and $p_{\mathrm{r}}$, respectively. Notice that, once the specification and pre-control limits are fixed, these probabilities depend only on the underlying distribution. The probability of qualifying the process will be denoted by $p_{\mathrm{Q}}$, which depends on $p_{\mathrm{g}}, p_{\mathrm{y}}$ and $p_{\mathrm{r}}$, and on the concrete qualification procedure.

Finally, the number of units to be sampled in order to finish the qualification stage of pre-control will be represented by $n$. As already highlighted in Section 1.1.1, $n$ is a random variable and is not initially upper bounded. This statement is valid for classical pre-control and also for most of the alternatives proposed in the literature, the work by Družovec et al. ${ }^{15}$ being the only exception. As in the case of $p_{\mathrm{Q}}$, the probability distribution of $n$ depends on $p_{\mathrm{g}}, p_{\mathrm{y}}$ and $p_{\mathrm{r}}$ and on the concrete qualification procedure.

From now, we will use the term pre-control to refer to the qualification stage of the technique, as it is the only stage which we are going to deal with in this paper.

\subsection{Pre-control as a test on the process mean}

Pre-control, like other quality control tools such as control charts, can be regarded as a statistical technique for testing hypotheses on the parameters of the underlying distribution (although this has been a matter of some controversy - see $^{19}$, for example). In particular, it can be seen as a test on the deviation of the real mean of the process $\mu$ with respect to the target value $\mu_{0}$, that is:

$$
\left\{\begin{array}{l}
H_{0}: \delta=0 \\
H_{1}: \delta>0
\end{array},\right.
$$


where $\delta$ represents the mean shift in sigma units, relative mean shift size or standardized mean shift (in absolute value) which is:

$$
\delta=\frac{\left|\mu_{0}-\mu\right|}{\sigma_{0}} .
$$

More precisely, the test would work as follows: If pre-control finishes qualifying the process, then the null hypothesis $H_{0}$ is not rejected, that is, the process is assumed to be centered or stable; otherwise, the mean of the process is considered to be different from the target.

According to this methodology, the probability of not rejecting $H_{0}$ as a function of $\delta$ is equal to the probability of qualifying the process (see equation (8) in Section 3 later, for generalized pre-control).

This approach (which supposes that it is ignored whether the process is centered or not) implies that an estimate of the actual standard deviation of the process or, equivalently, the value for $C_{p}$ or an estimation of it is known. Notice again that, unlike its original purpose, pre-control is considered here as a tool to detect changes in the process mean, as it actually happens in most of the attempts to improve this technique existing in the literature (see Section 1.2). Therefore, there is no contradiction in needing (an estimation of) the value of $C_{\mathrm{p}}$.

In this paper we consider pre-control as a test on the process mean. We also assume that the standard deviation of the process is under control and is equal to its target value $\sigma_{0}$. As has been previously said (Section 1.1.1), classical pre-control shows poor performance when used for this purpose, under certain circumstances. In Section 3 generalized pre-control is shown to overcome this issue.

Given a concrete pre-control procedure on a process with a certain known potential capability $C_{\mathrm{p}}$, the probabilities $p_{\mathrm{g}}, p_{\mathrm{y}}, p_{\mathrm{r}}$ and $p_{\mathrm{Q}}$, as well as the distribution of the random variable $n$, depend only on the value of $\mu$ or, equivalently, on the relative shift of the process mean $\delta$. This is why, in the following, we will express these quantities as functions of $\delta$-that is: $p_{\mathrm{g}}(\delta), n(\delta)$, for example. 


\section{Generalized pre-control}

Next we describe the proposal by San Matías et al. ${ }^{16}$, which we will call generalized pre-control (the authors do not give it a specific name). San Matías et al. suggest making the following parameters of pre-control flexible:

- $k$, the number of consecutive green units to be sampled in order to qualify the process,

- $t$, the number of consecutive yellow units to be sampled in order to stop the process, and

- $W_{G}$, the width of the green zone.

The value of the parameter $W_{\mathrm{G}}$ affects the span of the green and yellow zones, and hence the values of the probabilities $p_{\mathrm{g}}$ and $p_{\mathrm{y}}$. The values of $k$ and $t$ directly influence the qualification procedure.

Actually, San Matías et al. use a scale-independent parameter called $\lambda$ instead of $W_{G}$, which is defined as the ratio between the width of the specification range and half the width of the green zone:

$$
\lambda=\frac{\mathrm{USL}-\mathrm{LSL}}{W_{\mathrm{G}} / 2}, \quad \lambda \geq 2 ;
$$

in other words, it represents the number of times that half the green zone is contained in the specification range (so, in classical pre-control $\lambda=4$ ). From now on, we will call a concrete setting of the so-called pre-control parameters $k, t$ and $\lambda$ a pre-control plan.

In this way, pre-control can be customized in order to better meet the user's requirements, in terms of the false alarm rate and the probability of (not) detecting a given undesirable (large) deviation in the process mean.

More precisely, using a recursive strategy, San Matías et al. develop a concrete expression for the probability of qualifying the process as a function of the relative mean shift size $\delta$, given a concrete pre-control plan. 
After adapting it to our notation, the expression is as follows:

$$
p_{\mathrm{Q}}(\delta)=p_{\mathrm{g}}(\delta)^{k} \frac{\sum_{j=0}^{t-1} p_{\mathrm{y}}(\delta)^{j}}{1-\sum_{j=1}^{t-1} p_{\mathrm{y}}(\delta)^{j} \sum_{i=1}^{k-1} p_{\mathrm{g}}(\delta)^{i}},
$$

which can also be expressed as

$$
p_{\mathrm{Q}}(\delta)=\frac{p_{\mathrm{g}}(\delta)^{k}\left(1-p_{\mathrm{g}}(\delta)\right)\left(1-p_{\mathrm{y}}(\delta)^{t}\right)}{\left(1-p_{\mathrm{g}}(\delta)\right)\left(1-p_{\mathrm{y}}(\delta)\right)-\left(p_{\mathrm{g}}(\delta)-p_{\mathrm{g}}(\delta)^{k}\right)\left(p_{\mathrm{y}}(\delta)-p_{\mathrm{y}}(\delta)^{t}\right)}
$$

using

$$
\sum_{i=0}^{m-1} r^{i}=\frac{1-r^{m}}{1-r} \quad \forall r: r \neq 1
$$

For the normal two-sided symmetric case that we are dealing with, the probabilities $p_{\mathrm{g}}(\delta)$ and $p_{\mathrm{y}}(\delta)$ can be calculated as ${ }^{16}$ :

$$
\begin{aligned}
& p_{\mathrm{g}}(\delta)=\Phi\left(\delta+\frac{6 C_{\mathrm{p}}}{\lambda}\right)-\Phi\left(\delta-\frac{6 C_{\mathrm{p}}}{\lambda}\right) \\
& p_{\mathrm{y}}(\delta)=\Phi\left(\delta+3 C_{\mathrm{p}}\right)-\Phi\left(\delta-3 C_{\mathrm{p}}\right)-p_{\mathrm{g}}(\delta)
\end{aligned}
$$

and therefore

$$
p_{\mathrm{r}}(\delta)=1-\Phi\left(\delta+3 C_{\mathrm{p}}\right)+\Phi\left(\delta-3 C_{\mathrm{p}}\right),
$$

where $\Phi$ represents the cumulative distribution function of a standard normal variable. Notice that, as said before, an estimation of $C_{\mathrm{p}}$ is needed.

If we consider pre-control as a test on the relative shift of the process mean $\delta$ (see Section 2.2), then expressions for the probability of type I error or false alarm rate $\alpha$ and the probability of type II error $\beta\left(\delta^{*}\right)$ for a given deviation $\delta^{*}$ (also known as miss rate) can easily be obtained:

$$
\alpha=1-p_{\mathrm{Q}}(0)
$$




$$
\beta\left(\delta^{*}\right)=p_{\mathrm{Q}}\left(\delta^{*}\right),
$$

where $p_{\mathrm{Q}}(\delta)$ is calculated via (8).

Given a concrete upper bound $\alpha^{*}$ for the false alarm rate and a concrete upper bound $\beta^{*}$ for the probability of not detecting a certain unacceptable deviation $\delta^{*}$, San Matías et al. describe a methodology for finding precontrol plans which meet those requirements, that is, which verify

$$
\left.\begin{array}{rl}
\alpha & \leq \alpha^{*} \\
\beta\left(\delta^{*}\right) & \leq \beta^{*}
\end{array}\right\},
$$

and show it through a numerical example.

As far as we know, the idea of adapting the width of the green zone of pre-control in order to meet some given requirements first appeared in Steiner ${ }^{14}$, as highlighted in Section 1.2. In addition, something similar to parametrizing the number of consecutive green or yellow units already appeared in Salvia ${ }^{20}$ (we will refer to this article again in appendix A) and also in Salvia ${ }^{21}$ regarding stoplight control. The term stoplight control is usually considered as a synonym for pre-control, although the version handled by Salvia ${ }^{21}$ shows a quite different acceptance scheme. However, to the best of our knowledge, San Matías et al. ${ }^{16}$ are the first to provide explicit equations for the general case and a concrete procedure to determine valid settings of the pre-control parameters with regards to some given requirements, instead of look-up tables for a selection of cases.

Therefore, as a conclusion, generalized pre-control succeeds in improving the performance of the classical technique and adapting it to the user's requirements in terms of the false alarm rate and the ability to detect unacceptable deviations, when considered as a test on the stability of the process mean.

\section{The Optimal Pre-control model}

As has been shown in Section 3, generalized pre-control provides the user with a concrete procedure to obtain a pre-control plan which meets 
his/her requirements, but also lets him/her decide which plan to choose, as several possible ones normally exist - an uncountable amount of them, in fact.

Instead of leaving the decision to the user, we propose applying an optimization approach to generalized pre-control, so that the best precontrol plan according to one given criterion is obtained in an automatic way, from among those verifying the requirements given by (16).

More precisely, in this paper we suggest selecting the combination of the (generalized) pre-control parameters which minimizes the expected sample size when the process is under control, that is, the one that minimizes $E[n(0)]$. This implies solving a Mathematical Programming model, the outline of which would be the following:

$$
\left.\begin{array}{lrl}
\text { Min } & E[n(0)] & \\
\text { s.t.: } & \alpha & \leq \alpha^{*} \\
& \beta\left(\delta^{*}\right) & \leq \beta^{*} \\
& k, t \geq 1, \lambda & \geq 2 \\
k, t \in \mathbb{Z}, \lambda & \in \mathbb{R}
\end{array}\right\} .
$$

Explicit expressions for the left-hand sides of the constraints in model (17) $\alpha$ and $\beta\left(\delta^{*}\right)$, respectively, can be derived based on what was shown in Section 3. Specifically, from (14), (15) and (9) it follows that

$$
\begin{gathered}
\alpha=\frac{p_{\mathrm{g}}(0)^{k}\left(1-p_{\mathrm{g}}(0)\right)\left(1-p_{\mathrm{y}}(0)^{t}\right)}{\left(1-p_{\mathrm{g}}(0)\right)\left(1-p_{\mathrm{y}}(0)\right)-\left(p_{\mathrm{g}}(0)-p_{\mathrm{g}}(0)^{k}\right)\left(p_{\mathrm{y}}(0)-p_{\mathrm{y}}(0)^{t}\right)}, \\
\beta\left(\delta^{*}\right)=\frac{p_{\mathrm{g}}\left(\delta^{*}\right)^{k}\left(1-p_{\mathrm{g}}\left(\delta^{*}\right)\right)\left(1-p_{\mathrm{y}}\left(\delta^{*}\right)^{t}\right)}{\left(1-p_{\mathrm{g}}\left(\delta^{*}\right)\right)\left(1-p_{\mathrm{y}}\left(\delta^{*}\right)\right)-\left(p_{\mathrm{g}}\left(\delta^{*}\right)-p_{\mathrm{g}}\left(\delta^{*}\right)^{k}\right)\left(p_{\mathrm{y}}\left(\delta^{*}\right)-p_{\mathrm{y}}\left(\delta^{*}\right)^{t}\right)},
\end{gathered}
$$

where $p_{\mathrm{g}}(\delta)$ and $p_{\mathrm{y}}(\delta)$ are calculated as in (11) and (12), respectively.

An explicit expression for the objective function of model (17) $E[n(0)]$ is 
also needed. The following equivalences can be proven (see appendix A):

$$
\begin{aligned}
E[n(0)] & =\frac{\sum_{i=0}^{k-1} p_{\mathrm{g}}(0)^{i} \sum_{j=0}^{t-1} p_{\mathrm{y}}(0)^{j}}{1-\sum_{i=1}^{k-1} p_{\mathrm{g}}(0)^{i} \sum_{j=1}^{t-1} p_{\mathrm{y}}(0)^{j}} \\
& =\frac{\left(1-p_{\mathrm{g}}(0)^{k}\right)\left(1-p_{\mathrm{y}}(0)^{t}\right)}{\left(1-p_{\mathrm{g}}(0)\right)\left(1-p_{\mathrm{y}}(0)\right)-\left(p_{\mathrm{g}}(0)-p_{\mathrm{g}}(0)^{k}\right)\left(p_{\mathrm{y}}(0)-p_{\mathrm{y}}(0)^{t}\right)},
\end{aligned}
$$

and even this more compact one:

$$
E[n(0)]=\left(\left(\sum_{i=0}^{k-1} p_{\mathrm{g}}(0)^{i}\right)^{-1}+\left(\sum_{j=0}^{t-1} p_{\mathrm{y}}(0)^{j}\right)^{-1}-1\right)^{-1} .
$$

Therefore, according to (17), (21), (18) and (19), given a process with known potential capability $C_{\mathrm{p}}$ to be monitored and given the problem parameters $\left.\alpha^{*} \in\right] 0,1\left[, \beta^{*} \in\right] 0,1\left[\right.$ and $\delta^{*}>0$, the question of determining the setting of the pre-control parameters $k, t$ and $\lambda$ which minimizes the expected sample size of the qualification procedure when the process is centered, while ensuring a maximum false alarm rate of $\alpha^{*}$ and detecting a mean shift of at least $\delta^{*}$ with probability greater than or equal to $1-\beta^{*}$ can be answered by solving the following mathematical model:

$$
\left.\begin{array}{cc}
\operatorname{Min} \quad \frac{\left(1-p_{\mathrm{g}}(0)^{k}\right)\left(1-p_{\mathrm{y}}(0)^{t}\right)}{\left(1-p_{\mathrm{g}}(0)\right)\left(1-p_{\mathrm{y}}(0)\right)-\left(p_{\mathrm{g}}(0)-p_{\mathrm{g}}(0)^{k}\right)\left(p_{\mathrm{y}}(0)-p_{\mathrm{y}}(0)^{t}\right)} \\
\text { s.t.: } \quad \frac{p_{\mathrm{g}}(0)^{k}\left(1-p_{\mathrm{g}}(0)\right)\left(1-p_{\mathrm{y}}(0)^{t}\right)}{\left(1-p_{\mathrm{g}}(0)\right)\left(1-p_{\mathrm{y}}(0)\right)-\left(p_{\mathrm{g}}(0)-p_{\mathrm{g}}(0)^{k}\right)\left(p_{\mathrm{y}}(0)-p_{\mathrm{y}}(0)^{t}\right)} \leq \alpha^{*} \\
\frac{p_{\mathrm{g}}\left(\delta^{*}\right)^{k}\left(1-p_{\mathrm{g}}\left(\delta^{*}\right)\right)\left(1-p_{\mathrm{y}}\left(\delta^{*}\right)^{t}\right)}{\left(1-p_{\mathrm{g}}\left(\delta^{*}\right)\right)\left(1-p_{\mathrm{y}}\left(\delta^{*}\right)\right)-\left(p_{\mathrm{g}}\left(\delta^{*}\right)-p_{\mathrm{g}}\left(\delta^{*}\right)^{k}\right)\left(p_{\mathrm{y}}\left(\delta^{*}\right)-p_{\mathrm{y}}\left(\delta^{*}\right)^{t}\right)} \leq \beta^{*} \\
k, t \geq 1, \lambda \geq 2, \quad k, t \in \mathbb{Z}, \lambda \in \mathbb{R}
\end{array}\right\} .
$$

We will refer to model (23) as problem OPC $\left(\alpha^{*}, \beta^{*}, \delta^{*}\right)$ or simply problem OPC (for optimal pre-control). Due to its structure, the problem OPC is a mixed-integer nonlinear problem, which can be solved using appropriate 
Optimization software.

As a guide for practitioners, we summarize our proposal through the following procedure:

Step 1. Determining the model parameters. Estimate $C_{\mathrm{p}}$ and select the required values for $\alpha^{*}, \beta^{*}$ and $\delta^{*}$. The value for $\delta^{*}$ can be also derived through equation (13) by selecting a value for an unacceptable proportion of nonconforming units $p_{\mathrm{r}}\left(\delta^{*}\right)$.

Step 2. Solving the optimization model. Solve the problem $\operatorname{OPC}\left(\alpha^{*}, \beta^{*}\right.$, $\left.\delta^{*}\right)(23)$ to optimality.

Step 3. If no solution was found, which means that the requirements were too tight, then go to Step 1 and relax the requirements on the probabilities $\alpha$ and /or $\beta\left(\delta^{*}\right)$. Otherwise, go to Step 4 .

Step 4. Communicating the optimal plan. The optimal pre-control plan $\left(k^{\star}, t^{\star}, \lambda^{\star}\right)$ is the one obtained by solving the model in Step 2. This means that

- the number of consecutive greens to be observed in order to qualify the process is $k^{\star}$,

- the number of consecutive yellows to be observed in order to stop and revise the process is $t^{\star}$, and

- the width of the green area has to be fixed to $W_{\mathrm{G}}=\frac{\text { USL-LSL }}{\lambda^{\star} / 2}$ (according to (7)).

- Furthermore, the limits of the green zone can be calculated using (2) and (3).

- The expected sample size to be needed by the optimal plan when the process is centered is given by (21).

- Additional information such as the actual false alarm and miss rates for this plan can be computed with (14) and (15). 


\section{Numerical experiments}

From the Optimization point of view which we have just introduced, generalized pre-control can now be seen as a tool for finding feasible solutions to the Mathematical Programming problem (23). For example, consider the OPC problem (23) with requirements $\alpha^{*}=0.005, \beta^{*}=0.10$ and $\delta^{*}=1.5$ for a process with $C_{\mathrm{p}}=1.3333$. Solving (23) to optimality in this case, we get the following solution: $(k=7, t=4, \lambda=5.28417)$ -that is, a green zone width of $W_{\mathrm{G}}=(\mathrm{USL}-\mathrm{LSL}) /(5.28417 / 2)=$ $37.85 \%$ (USL - LSL), according to (7)—, that gives $E[n(0)]=12.6503$ units, while the first solution provided by the algorithm of generalized precontrol by San Matías et al. ${ }^{16}(k=5, t=6, \lambda=7.27011)$ would lead to an expected sample size of 14.1723 units, according to (20). Figures 2 and 3 show the operating characteristic curves of the classical and the optimal pre-control plans for this example, as well as the resulting expected sample size as a function of the relative mean shift size $\delta$ in both cases.

As a way to assess the effectiveness of our proposal, we have solved to optimality a set of 36 instances of problem OPC (23), resulting from the following combination: $C_{\mathrm{p}} \in\{1.2,1.3333,1.4,1.5\} \times$ $\alpha^{*} \in\{0.27 \%, 0.5 \%, 1 \%\} \times \beta^{*} \in\{0.5 \%, 5 \%, 10 \%\}$, with $\delta^{*}$ such that $p_{\mathrm{r}}\left(\delta^{*}\right)=2 \%$ (see equation (13)).

Table 1 shows the results obtained for these instances. As it can be seen, some of the examples (numbered from 1 to 5 ) were found to be infeasible. This occurred because the constraints on $\alpha$ and $\beta\left(\delta^{*}\right)$ where mutually incompatible in those instances, all of them associated with the lowest capability value in the experiment $\left(C_{p}=1.2\right)$. Aiming at a better comparison, table 2 shows the values of $\alpha, \beta\left(\delta^{*}\right)$ and $E[n(0)]$ which would be obtained if classical pre-control was applied to the same 36 instances. 


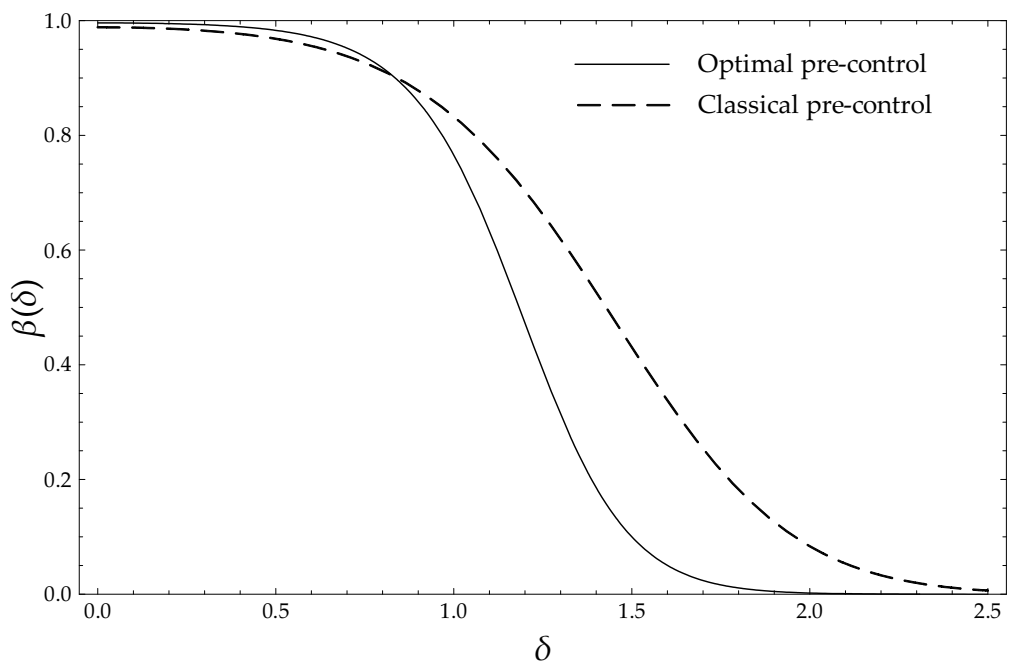

Figure 2: Operating characteristic curves for the classical qualification procedure and the optimal pre-control plan obtained for the example in Section 5.

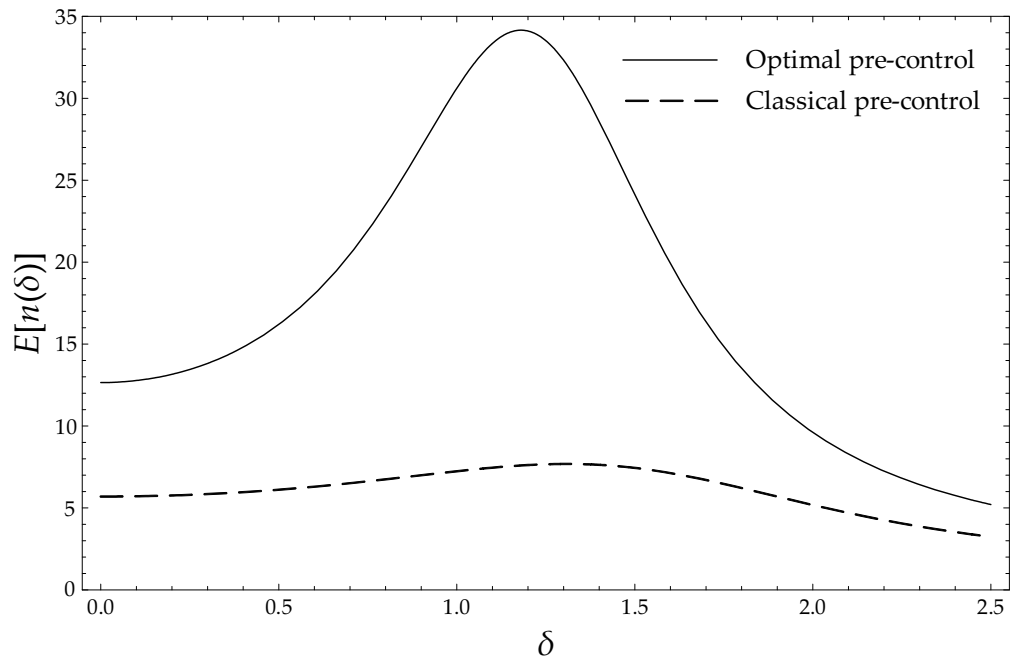

Figure 3: Expected sample size for the classical qualification procedure and the optimal pre-control plan obtained for the example in Section 5. 


\section{Discussion and conclusions}

Pre-control has been labeled a "naive" and even "folkloric" tool by some authors $^{22,23}$, mainly because it has been sometimes wrongly promoted as an alternative to Shewhart's control charts, together with the relative lack of research on it. Some of its weaknesses have been addressed in previous works, such as those by Steiner ${ }^{14}$ and San Matías et al. ${ }^{16}$.

Moreover, quality control tools based on counts or on yes/no attributes are less efficient than using (continuous) variables data, in general terms, due to the information and accuracy loss. However, it is also true that gauging (that is, the fact of measuring whether units fall within certain -narrow- limits or not, as in pre-control) is considered to be a good alternative in certain contexts, mainly because of its simplicity (see ${ }^{24}$, for instance).

In this paper we have suggested applying an Optimization approach to the design of the qualification phase of pre-control. A Mathematical Programming model has been proposed, aimed at optimally determining the values for the pre-control parameters, as defined in San Matías et al. ${ }^{16}$.

The so-called optimal pre-control makes it possible to adjust the qualification procedure in such a way that required respective upper bounds for the false alarm rate and the probability of not detecting an undesirable deviation of the process are taken into account, while minimizing the average number of units to be sampled when the process is under control, the process mean being the monitored parameter. The cost of this improvement is often an eventual increase in the required sample size in comparison with the classical technique, as it normally occurs in statistical tools for hypothesis testing. All this has been shown through a numerical experiment.

A procedure summarizing all the steps to be performed in order to put optimal pre-control into practice has been presented. Furthermore, the results of the numerical experiment collected in table 1 can be also regarded as a look-up table for quick selections of optimal plans.

A formal comparison with the work of Družovec et al. ${ }^{15}$ and Cox 
et al. ${ }^{18}$ may not make sense, because of the methodological differences and due to the fact that, as our proposal allows for more degrees of freedom (in the sense that more parameters can be adjusted), and also introduces an Optimization approach, it is able to better adapt to a wider range of user's requirements.

Unlike classical pre-control, the approach that has been introduced in this paper assumes that the process is normally distributed and that its natural variability can be known or estimated, in the same way as other recent proposals aimed at improving the classical technique (see ${ }^{18}$, for instance). However, the expressions for the functions involved in the outline of the mathematical model (17) - that is, the objective function (20) and the left-hand sides of the constraints (18) and (19) - are also adequate for other underlying distributions, even in contexts in which the quality characteristic is not continuous or even not quantitative, as far as the concept of shift with regards to a target still applies and values for $p_{\mathrm{g}}$ and $p_{\mathrm{y}}$ can be calculated as a function of that shift.

In conclusion, optimal pre-control is a useful and powerful tool for set-up approval in manufacturing processes. It addresses some of the issues of the classical technique by applying a Mixed-Integer Nonlinear Programming approach, while still remaining simple, fast and effective.

\section{Appendix A. Derivation of an expression for $E[n(\delta)]$}

In this Section an analytic expression for the expected sample size $E[n(\delta)]$ of the qualification procedure in generalized pre-control is derived. The result is valid for any value of $\delta \geq 0$, and in particular for $\delta=0$, which is the case considered in the objective function of problem OPC (23).

The methodology we follow is simply founded on basic probability theory and linear system solving, and is totally equivalent to the Markov chain approach used by Salvia ${ }^{20}$, who shows how to calculate both the probability of pre-control qualifying a process $p_{\mathrm{Q}}(\delta)$ and the expected number of units to be sampled $E[n(\delta)]$, for any given pre-control plan, and applies this to several concrete examples. However, the author does 
not give explicit equations for the general case - that is to say, expressions to directly calculate $p_{\mathrm{Q}}(\delta)$ and $E[n(\delta)]$ as functions of $k$ and $t$.

We start by defining the following states, which the qualification procedure goes through:

- State 0 or initial state, prior to the start of sampling.

- State F or final state: represents the end of the qualification procedure. It can be reached after obtaining $k$ consecutive greens, or $t$ consecutive yellows or 1 red.

- State $i \mathrm{G}$, when the last sampled unit is green and we have already accumulated a series of $i$ consecutive green units $(i=1, \ldots, k-1)$.

- State $j \mathrm{Y}$, when the last sampled unit is yellow and we have already accumulated a series of $j$ consecutive yellow units $(j=1, \ldots, t-1)$.

Notice that the system is always in one of these $k+t$ different states.

We denote the number of units to be sampled in order to reach state $b$ starting from state $a$ in a process with standardized mean shift $\delta$ by $n_{a, b}(\delta)$. In particular, $n_{a, \mathrm{~F}}(\delta)$ is the number of units which it is necessary to draw in order to reach the final state when the process is in state $a$. We call the expected value of this random variable $x_{a}^{\delta}$, that is:

$$
x_{a}^{\delta}=E\left[n_{a, \mathrm{~F}}(\delta)\right] .
$$

According to the notation which was introduced in Section 2.1, $n(\delta)=$ $n_{0, \mathrm{~F}}(\delta)$, and therefore $E[n(\delta)]=x_{0}^{\delta}$, which is the value we are interested in.

The discrete random variable $n_{0, \mathrm{~F}}(\delta)$ has the following distribution function:

$$
n_{0, \mathrm{~F}}(\delta)= \begin{cases}1+n_{1 \mathrm{G}, \mathrm{F}}(\delta) & \text { with probability } p_{\mathrm{g}}(\delta) \\ 1+n_{1 \mathrm{Y}, \mathrm{F}}(\delta) & \text { with probability } p_{\mathrm{y}}(\delta) \\ 1 & \text { con probabilidad } p_{\mathrm{r}}(\delta)\end{cases}
$$


We calculate the expected value of expression (25) to obtain

$$
x_{0}^{\delta}=\left(1+x_{1 \mathrm{G}}^{\delta}\right) p_{\mathrm{g}}(\delta)+\left(1+x_{1 \mathrm{Y}}^{\delta}\right) p_{\mathrm{y}}(\delta)+p_{\mathrm{r}}(\delta),
$$

which can be rewritten as

$$
x_{0}^{\delta}=1+p_{\mathrm{g}}(\delta) x_{1 \mathrm{G}}^{\delta}+p_{\mathrm{y}}(\delta) x_{1 \mathrm{Y}}^{\delta}
$$

using $p_{\mathrm{g}}(\delta)+p_{\mathrm{y}}(\delta)=1-p_{\mathrm{r}}(\delta)$.

In general:

$$
n_{i \mathrm{G}, \mathrm{F}}(\delta)= \begin{cases}1+n_{(i+1) \mathrm{G}, \mathrm{F}}(\delta) & \text { with probability } p_{\mathrm{g}}(\delta) \\ 1+n_{1 \mathrm{Y}, \mathrm{F}}(\delta) & \text { with probability } p_{\mathrm{y}}(\delta) \\ 1 & \text { with probability } p_{\mathrm{r}}(\delta)\end{cases}
$$

for $i=1, \ldots, k-2$, and

$$
n_{(k-1) \mathrm{G}, \mathrm{F}}(\delta)=\left\{\begin{array}{ll}
1+n_{1 \mathrm{Y}, \mathrm{F}}(\delta) & \text { with probability } p_{\mathrm{y}}(\delta) \\
1 & \text { with probability } p_{\mathrm{g}}(\delta)+p_{\mathrm{r}}(\delta)
\end{array} .\right.
$$

Similarly, for $j=1, \ldots, t-2$ :

$$
n_{j \mathrm{Y}, \mathrm{F}}(\delta)= \begin{cases}1+n_{1 \mathrm{G}, \mathrm{F}}(\delta) & \text { with probability } p_{\mathrm{g}}(\delta) \\ 1+n_{(j+1) \mathrm{Y}, \mathrm{F}}(\delta) & \text { with probability } p_{\mathrm{y}}(\delta) \\ 1 & \text { con probabilidad } p_{\mathrm{r}}(\delta)\end{cases}
$$

and

$$
n_{(t-1) \mathrm{Y}, \mathrm{F}}(\delta)=\left\{\begin{array}{ll}
1+n_{1 \mathrm{G}, \mathrm{F}}(\delta) & \text { with probability } p_{\mathrm{g}}(\delta) \\
1 & \text { with probability } p_{\mathrm{y}}(\delta)+p_{\mathrm{r}}(\delta)
\end{array} .\right.
$$

Proceeding in a similar way to the operation with $n_{0, \mathrm{~F}}(\delta)$, we calculate 
the expected values of expressions (28), (29), (30) and (31):

$$
\begin{aligned}
x_{i \mathrm{G}}^{\delta} & =1+p_{\mathrm{g}}(\delta) x_{(i+1) \mathrm{G}}^{\delta}+p_{\mathrm{y}}(\delta) x_{1 \mathrm{Y}}^{\delta}, \quad \text { for } i=1, \ldots, k-2, \\
x_{(k-1) \mathrm{G}}^{\delta} & =1+p_{\mathrm{y}}(\delta) x_{1 \mathrm{Y}}^{\delta}, \\
x_{j \mathrm{Y}}^{\delta} & =1+p_{\mathrm{g}}(\delta) x_{1 \mathrm{G}}^{\delta}+p_{\mathrm{y}}(\delta) x_{(j+1) \mathrm{Y}}^{\delta}, \quad \text { for } j=1, \ldots, t-2, \\
x_{(t-1) \mathrm{Y}}^{\delta} & =1+p_{\mathrm{g}}(\delta) x_{1 \mathrm{G}}^{\delta} .
\end{aligned}
$$

Combining (27), (32), (33), (34) and (35) we obtain the following linear system with $k+t-1$ equations and unknowns:

$$
\begin{aligned}
x_{0}^{\delta} & =1+p_{\mathrm{g}}(\delta) x_{1 \mathrm{G}}^{\delta}+p_{\mathrm{y}}(\delta) x_{1 \mathrm{Y}}^{\delta} \\
x_{1 \mathrm{G}}^{\delta} & =1+p_{\mathrm{g}}(\delta) x_{2 \mathrm{G}}^{\delta}+p_{\mathrm{y}}(\delta) x_{1 \mathrm{Y}}^{\delta} \\
& \cdots \\
x_{(k-2) \mathrm{G}}^{\delta} & =1+p_{\mathrm{g}}(\delta) x_{(k-1) \mathrm{G}}^{\delta}+p_{\mathrm{y}}(\delta) x_{1 \mathrm{Y}}^{\delta} \\
x_{(k-1) \mathrm{G}}^{\delta} & =1+p_{\mathrm{y}}(\delta) x_{1 \mathrm{Y}}^{\delta} \\
x_{1 \mathrm{Y}}^{\delta} & =1+p_{\mathrm{g}}(\delta) x_{1 \mathrm{G}}^{\delta}+p_{\mathrm{y}}(\delta) x_{2 \mathrm{Y}}^{\delta} \\
& \cdots \\
x_{(t-2) \mathrm{Y}}^{\delta} & =1+p_{\mathrm{g}}(\delta) x_{1 \mathrm{G}}^{\delta}+p_{\mathrm{y}}(\delta) x_{(t-1) \mathrm{Y}}^{\delta} \\
x_{(t-1) \mathrm{Y}}^{\delta} & =1+p_{\mathrm{g}}(\delta) x_{1 \mathrm{G}}^{\delta}
\end{aligned}
$$

We obtain the following expressions for $x_{1 \mathrm{G}}^{\delta}$ and $x_{1 \mathrm{Y}}^{\delta}$ by solving (36):

$$
\begin{aligned}
& x_{1 \mathrm{G}}^{\delta}=\frac{\sum_{i=0}^{k-2} p_{\mathrm{g}}(\delta) \sum_{j=0}^{i-1} p_{\mathrm{y}}(\delta)^{j}}{1-p_{\mathrm{g}}(\delta) p_{\mathrm{y}}(\delta) \sum_{i=0}^{k-2} p_{\mathrm{g}}(\delta)^{i} \sum_{j=0}^{t-2} p_{\mathrm{y}}(\delta)^{j}}, \\
& x_{1 \mathrm{Y}}^{\delta}=\left(1+p_{\mathrm{g}}(\delta) x_{1 G}^{\delta}\right) \sum_{j=0}^{t-2} p_{\mathrm{y}}(\delta)^{j} .
\end{aligned}
$$

Finally, we obtain an expression for $E[n(\delta)]$ by replacing (37) and (38) 
in (27):

$$
\begin{aligned}
E[n(\delta)]= & x_{0}^{\delta} \\
= & \sum_{j=0}^{t-1} p_{\mathrm{y}}(\delta)^{j}\left(1+\frac{p_{\mathrm{g}}(\delta) \sum_{i=0}^{k-2} p_{\mathrm{g}}(\delta)^{i} \sum_{j=0}^{t-1} p_{\mathrm{y}}(\delta)^{j}}{1-p_{\mathrm{g}}(\delta) \sum_{i=0}^{k-2} p_{\mathrm{g}}(\delta)^{i} p_{\mathrm{y}}(\delta) \sum_{j=0}^{t-2} p_{\mathrm{y}}(\delta)^{j}}\right) \\
= & \frac{\sum_{i=0}^{k-1} p_{\mathrm{g}}(\delta)^{i} \sum_{j=0}^{t-1} p_{\mathrm{y}}(\delta)^{j}}{1-\sum_{i=1}^{k-1} p_{\mathrm{g}}(\delta)^{i} \sum_{j=1}^{t-1} p_{\mathrm{y}}(\delta)^{j}}
\end{aligned}
$$

If we solve (36) for the particular cases where $k=1$ or $t=1$, we obtain

$$
x_{0}=\sum_{i=0}^{k-1} p_{\mathrm{g}}(\delta)^{i} \sum_{j=0}^{t-1} p_{\mathrm{y}}(\delta)^{j}
$$

which can be reduced to the general expression (39) by using (40):

$$
\sum_{i=1}^{m-1} r^{i}=\sum_{i=0}^{m-1} r^{i}-1 \quad \forall r
$$

The expression (39) can be simplified using (10):

$$
E[n(\delta)]=\frac{\left(1-p_{\mathrm{g}}(\delta)^{k}\right)\left(1-p_{\mathrm{y}}(\delta)^{t}\right)}{\left(1-p_{\mathrm{g}}(\delta)\right)\left(1-p_{\mathrm{y}}(\delta)\right)-\left(p_{\mathrm{g}}(\delta)-p_{\mathrm{g}}(\delta)^{k}\right)\left(p_{\mathrm{y}}(\delta)-p_{\mathrm{y}}(\delta)^{t}\right)},
$$

and can also be expressed in a more compact form using (40):

$$
E[n(\delta)]=\frac{\sum_{i=0}^{k-1} p_{\mathrm{g}}(\delta)^{i} \sum_{j=0}^{t-1} p_{\mathrm{y}}(\delta)^{j}}{1-\left(\sum_{i=0}^{k-1} p_{\mathrm{g}}(\delta)^{i}-1\right)\left(\sum_{j=0}^{t-1} p_{\mathrm{y}}(\delta)^{j}-1\right)}
$$




$$
\begin{aligned}
& =\frac{\sum_{i=0}^{k-1} p_{\mathrm{g}}(\delta)^{i} \sum_{j=0}^{t-1} p_{\mathrm{y}}(\delta)^{j}}{\sum_{i=0}^{k-1} p_{\mathrm{g}}(\delta)^{i}+\sum_{j=0}^{t-1} p_{\mathrm{y}}(\delta)^{j}-\sum_{i=0}^{k-1} p_{\mathrm{g}}(\delta)^{i} \sum_{j=0}^{t-1} p_{\mathrm{y}}(\delta)^{j}} \\
& =\frac{1}{\sum_{i=0}^{k-1} p_{\mathrm{g}}(\delta)^{i}}+\frac{1}{\sum_{j=0}^{t-1} p_{\mathrm{y}}(\delta)^{j}}-1 \\
& =\left(\left(\sum_{i=0}^{k-1} p_{\mathrm{g}}(\delta)^{i}\right)^{-1}+\left(\sum_{j=0}^{t-1} p_{\mathrm{y}}(\delta)^{j}\right)^{-1}-1\right)^{-1} .
\end{aligned}
$$

\section{Acknowledgments}

The research by V. Giner-Bosch and M. Clemente-Císcar was partially supported by the Ministerio de Ciencia e Innovación of Spain under grant no. MTM2013-45381-P.

\section{References}

[1] Satterthwaite FE: A Simple, Effective Process Control Method, Report 54-1, Rath and Strong, Boston, MA, 1954.

[2] Shainin D, Shainin P: Pre-Control versus $\bar{X} \& R$ charting: continuous or immediate quality improvement? Quality Engineering , 1989; 1(4):419-429.

[3] Rahim MA, Al-Sultan KS: Some Contemporary Approaches to Optimization Models in Process Control, in Al-Sultan KS, Rahim MA (eds.), Optimization in Quality Control, Kluwer Academic Publishers, London, 1997; .

[4] Carlyle WM, Montgomery DC, Runger GC: Optimization Problems and Methods in Quality Control and Improvement. Journal of Quality Technology , 2000; 32(1):1-17. 
[5] Feng Q, Kapur KC: Quality control, in Ravindran AR (ed.), Operations Research Applications, CRC Press, Boca Raton, FL, chap. 2, 2009; .

[6] Woodall WH, Montgomery DC: Research Issues and Ideas in Statistical Process Control. Journal of Quality Technology , 1999; 31(4):376-386.

[7] Kethley RB, Peters MH: Extending economic design of $p$ charts to handle user specified constraints using a genetic algorithm. IIE Transactions , 2004; 36(9):855-864.

[8] García-Díaz JC, Aparisi F: Economic design of EWMA control charts using regions of maximum and minimum ARL. IIE Transactions , 2005; 37(11):1011-1021, doi:10.1080/07408170500232214.

[9] Chou CY, Chen $\mathrm{CH}$ : Economic design of variable sampling intervals $T^{2}$ control charts using genetic algorithms. Expert Systems with Applications , 2006; 30(2):233-242, doi:10.1016/j.eswa.2005.07.010.

[10] Vommi VB, Seetala MSN: A new approach to robust economic design of control charts. Applied Soft Computing , 2007; 7(1):211-228.

[11] García-Díaz JC, Aparisi F: Optimal parameters for CUSUM-FIR control charts for monitoring process mean. European Journal of Industrial Engineering , 2014; 8(1):69-89, doi:10.1504/EJIE.2014.059350.

[12] Salvia AA: Stoplight Control. Quality Progress , 1988; 21(9):39-42.

[13] Gruska GF, Heaphy MS: Stop Light Control - Revisited. American Society for Quality Control, Statistics Division Newsletter , 1991; 11(4):1112.

[14] Steiner SH: Pre-control and Some Simple Alternatives. Quality Engineering , 1997; 10(1):65-74, doi:10.1080/08982119708919110.

[15] Družovec M, Šostar A, Godina A: MPC process control procedure. Strojniški Vestnik-Journal of Mechanical Engineering , 1998; 44(1-2):29-34. 
[16] San Matías S, Jabaloyes J, Carrión A: Some modifications of the Classical Pre-control technique. Quality and Reliability Engineering International , 2004; 20(1):47-60, doi:10.1002/qre.542.

[17] Cox S, Garside JA, Kotsialos A: Set-up Dominated Low Volume Process Capability Estimation Using a Pre-Control Method, in Proceedings of the 1st International Through-Life Engineering Services Conference (TESConf 2012), Cranfield University Press, Shrivenham, 221-226.

[18] Cox S, Garside JA, Kotsialos A: Discrete-Event Simulation of Process Control in Low Volume High Value Industries, in Advances in Manufacturing Technology XXVII. Proceedings of the 11th International Conference on Manufacturing Research (ICMR 2013), Cranfield University Press, Cranfield, 599-604.

[19] Woodall WH: Controversies and contradictions in statistical process control. Journal of Quality Technology , 2000; 32(4):341-350.

[20] Salvia AA: Performance of PRE-Control Sampling Plans. Journal of Quality Technology , 1987; 19(2):85-89.

[21] Salvia AA: AOQ and AOQL for Stoplight Acceptance Sampling. Journal of Quality Technology , 1988; 20(3):157-161.

[22] Logothetis N: The theory of'Pre-Control': a serious method or a colourful naivity? Total Quality Management , 1990; 1(2):207-220.

[23] Ryan TP: Statistical Methods for Quality Improvement, John Wiley \& Sons, 3rd edn., 2011.

[24] Farnum N, Stanton L: Using Counts to Monitor a Process Mean. Journal of Quality Technology , 1986; 18(1):22-28. 


\begin{tabular}{|c|c|c|c|c|c|c|c|c|c|c|c|}
\hline \multirow{2}{*}{$\begin{array}{c}\text { Instance } \\
\text { no. }\end{array}$} & \multicolumn{4}{|c|}{ Problem parameters } & \multicolumn{5}{|c|}{ Optimal solution } & \multicolumn{2}{|c|}{ Additional info. } \\
\hline & $C_{\mathrm{p}}$ & $\alpha^{*}$ & $\beta^{*}$ & $\delta^{*}$ & $k$ & $t$ & $\lambda$ & $W_{\mathrm{G}}$ & $E[n(0)]$ & Slack $\alpha$ & Slack $\beta$ \\
\hline 1 & 1.2 & 0.0027 & 0.005 & 1.5462 & \multicolumn{5}{|c|}{ Infeasible } & - & - \\
\hline 2 & 1.2 & 0.0027 & 0.05 & 1.5462 & \multicolumn{5}{|c|}{ Infeasible } & - & - \\
\hline 3 & 1.2 & 0.0027 & 0.1 & 1.5462 & \multicolumn{5}{|c|}{ Infeasible } & - & - \\
\hline 4 & 1.2 & 0.005 & 0.005 & 1.5462 & \multicolumn{5}{|c|}{ Infeasible } & - & - \\
\hline 5 & 1.2 & 0.005 & 0.05 & 1.5462 & \multicolumn{5}{|c|}{ Infeasible } & - & - \\
\hline 6 & 1.2 & 0.005 & 0.1 & 1.5462 & 5 & 6 & 5.8621 & $34.12 \%$ & 11.1162 & 0.000503564 & 0 \\
\hline 7 & 1.2 & 0.01 & 0.005 & 1.5462 & 11 & 5 & 4.7667 & $41.96 \%$ & 27.8427 & 0.000220028 & 0 \\
\hline 8 & 1.2 & 0.01 & 0.05 & 1.5462 & 7 & 4 & 4.8458 & $41.27 \%$ & 13.0902 & 0.00185242 & 0 \\
\hline 9 & 1.2 & 0.01 & 0.1 & 1.5462 & 4 & 6 & 6.8731 & $29.10 \%$ & 10.2431 & 0.00202094 & 0 \\
\hline 10 & 1.3333 & 0.0027 & 0.005 & 1.9463 & 7 & 4 & 5.0959 & $39.25 \%$ & 11.8100 & 0.0000362568 & 30 \\
\hline 11 & 1.3333 & 0.0027 & 0.05 & 1.9463 & 4 & 5 & 5.8981 & $33.91 \%$ & 6.6121 & 0.00138719 & 0 \\
\hline 12 & 1.3333 & 0.0027 & 0.1 & 1.9463 & 3 & 5 & 6.4599 & $30.96 \%$ & 4.9612 & 0.000575501 & 0 \\
\hline 13 & 1.3333 & 0.005 & 0.005 & 1.9463 & 7 & 4 & 5.0959 & $39.25 \%$ & 11.8100 & 0.0023363 & 0 \\
\hline 14 & 1.3333 & 0.005 & 0.05 & 1.9463 & 4 & 4 & 5.6329 & $35.51 \%$ & 6.1923 & 0.00154942 & 0 \\
\hline 15 & 1.3333 & 0.005 & 0.1 & 1.9463 & 3 & 5 & 6.4599 & $30.96 \%$ & 4.9612 & 0.0028755 & 0 \\
\hline 16 & 1.3333 & 0.01 & 0.005 & 1.9463 & 6 & 4 & 5.5957 & $35.74 \%$ & 11.0913 & 0.00417719 & 0 \\
\hline 17 & 1.3333 & 0.01 & 0.05 & 1.9463 & 3 & 5 & 7.3682 & $27.14 \%$ & 5.9089 & 0.00258443 & 0 \\
\hline 18 & 1.3333 & 0.01 & 0.1 & 1.9463 & 3 & 4 & 6.0995 & $32.79 \%$ & 4.6126 & 0.00486982 & 0 \\
\hline 19 & 1.4 & 0.0027 & 0.005 & 2.1463 & 5 & 5 & 5.9710 & $33.50 \%$ & 8.6683 & 0.00171733 & 0 \\
\hline 20 & 1.4 & 0.0027 & 0.05 & 2.1463 & 3 & 5 & 6.5500 & $30.53 \%$ & 4.7553 & 0.00136512 & 0 \\
\hline 21 & 1.4 & 0.0027 & 0.1 & 2.1463 & 2 & 7 & 9.0620 & $22.07 \%$ & 3.9364 & 0.00082452 & 0 \\
\hline 22 & 1.4 & 0.005 & 0.005 & 2.1463 & 5 & 4 & 5.7825 & $34.59 \%$ & 8.2111 & 0.00156853 & 0 \\
\hline 23 & 1.4 & 0.005 & 0.05 & 2.1463 & 3 & 4 & 6.2352 & $32.08 \%$ & 4.4789 & 0.00118939 & 0 \\
\hline 24 & 1.4 & 0.005 & 0.1 & 2.1463 & 2 & 6 & 8.6201 & $23.20 \%$ & 3.7064 & 0.00170065 & 0 \\
\hline 25 & 1.4 & 0.01 & 0.005 & 2.1463 & 5 & 4 & 5.7826 & $34.59 \%$ & 8.2114 & 0.00656784 & 0 \\
\hline 26 & 1.4 & 0.01 & 0.05 & 2.1463 & 3 & 4 & 6.2352 & $32.08 \%$ & 4.4789 & 0.00618941 & 0 \\
\hline 27 & 1.4 & 0.01 & 0.1 & 2.1463 & 2 & 5 & 8.1312 & $24.60 \%$ & 3.4605 & 0.00386598 & 0 \\
\hline 28 & 1.5 & 0.0027 & 0.005 & 2.4463 & 4 & 4 & 5.9255 & $33.75 \%$ & 5.7056 & 0.00129319 & 0 \\
\hline 29 & 1.5 & 0.0027 & 0.05 & 2.4463 & 2 & 5 & 7.9180 & $25.26 \%$ & 3.1404 & 0.000122046 & 0 \\
\hline 30 & 1.5 & 0.0027 & 0.1 & 2.4463 & 2 & 4 & 6.4010 & $31.25 \%$ & 2.6026 & 0.00125861 & 0 \\
\hline 31 & 1.5 & 0.005 & 0.005 & 2.4463 & 3 & 5 & 7.9018 & $25.31 \%$ & 5.5329 & 0.000537003 & 0 \\
\hline 32 & 1.5 & 0.005 & 0.05 & 2.4463 & 2 & 5 & 7.9179 & $25.26 \%$ & 3.1404 & 0.00242227 & 0 \\
\hline 33 & 1.5 & 0.005 & 0.1 & 2.4463 & 2 & 3 & 5.9627 & $33.54 \%$ & 2.4638 & 0.000138451 & 0 \\
\hline 34 & 1.5 & 0.01 & 0.005 & 2.4463 & 4 & 3 & 5.6996 & $35.09 \%$ & 5.4289 & 0.00276868 & 0 \\
\hline 35 & 1.5 & 0.01 & 0.05 & 2.4463 & 2 & 4 & 7.4774 & $26.75 \%$ & 2.9589 & 0.0037167 & 0 \\
\hline 36 & 1.5 & 0.01 & 0.1 & 2.4463 & 2 & 3 & 5.9628 & $33.54 \%$ & 2.4638 & 0.0051383 & 0 \\
\hline
\end{tabular}

Table 1: Optimal pre-control plans for the examples considered in Section 5. The optimal width of the green area is expressed as a percentage of the width of the specification range. Slacks of each constraint with respect to the optimal solution are also provided as additional information. 


\begin{tabular}{ccccccc}
\hline \multirow{2}{*}{$\begin{array}{c}\text { Instance } \\
\text { no. }\end{array}$} & \multicolumn{2}{c}{ Problem parameters } & & \multicolumn{3}{c}{ Performance of classical pre-control } \\
\cline { 2 - 3 } \cline { 5 - 7 } & $C_{\mathrm{p}}$ & $\delta^{*}$ & & $\alpha$ & $\beta\left(\delta^{*}\right)$ & $E[n(0)]$ \\
\hline $1-9$ & 1.2 & 1.5462 & & 0.0310438 & 0.212529 & 6.09322 \\
$10-18$ & 1.3333 & 1.9463 & & 0.0116077 & 0.104600 & 5.69526 \\
$19-27$ & 1.4 & 2.1463 & & 0.00697237 & 0.0690471 & 5.54509 \\
$28-36$ & 1.5 & 2.4463 & & 0.00316906 & 0.0345236 & 5.37165 \\
\hline
\end{tabular}

Table 2: Results obtained with classical pre-control $(k=5, t=2, \lambda=4)$ in terms of $\alpha$, $\beta\left(\delta^{*}\right)$ and $E[n(0)]$ for the instances of the numerical experiment in Section 5. 